\title{
EL CONCEPTO “VIOLENCIA DE GÉNERO” EN LA PRENSA DIARIA NACIONAL ESPAÑOLA
}

\author{
Belén Zurbano Berenguer \\ bzurbano@us.es \\ Universidad de Sevilla
}

Recibido: 26-12-2011

Aceptado: 21-03-2012

\section{Resumen}

Con la aprobación de la Ley Orgánica 1/2004, de 28 de diciembre, de Medidas de Protección Integral contra la Violencia de Género, los medios de comunicación ven reconocido su inestimable valor como referentes a la hora de abordar las noticias de violencia de género. Sin embargo, también son de algún modo "recordados" por el legislador en su función social primigenia en cuanto a la responsabilidad que éstos tienen al construir la realidad que relatan. Esta investigación ha profundizado en la conceptualización que de este fenómeno de la violencia de género hacen los principales diarios de tirada nacional de nuestro país entendiendo que en la definición de las manifestaciones relatadas se encuentran muchos de los porqués de su ulterior tratamiento y selección temática. Para ello se ha seguido una metodología mixta basada en el análisis cualitativo desde una perspectiva próxima al Análisis del Discurso junto a técnicas de análisis de contenido cuantitativo. Las conclusiones principales apuntan a una falta de consenso en la terminología empleada y a una sobrerrepresentación de las muertes por violencia de género frente a otras manifestaciones de ésta que, además, en raras ocasiones quedan categorizadas como tal.

Palabras clave: Medios de comunicación, violencia de género, violencia doméstica, prensa nacional.

\begin{abstract}
When the Organic Act 1/2004 of 28 December on Integrated Protection Measures against Gender Violence (Nombre official que se encuentra en el Boletin official del Consejo de Europa) was approved, on December, the $28^{\text {th }}$, the media received the news as a reward for their work dealing with the genre-based violence everyday. However, they can't forget their role of reality makers for the eyes of society. This investigation study has approached the concept of this issue made by the main daily newspapers in Spain. According to their definition of "genre-based violence" and as a consequence of that, a lot of following theories can be also understood, such as the guidelines of news selection or the way of dealing with them. To get to this final resolution, the project was based on a methodology, which consisted in a quality analysis (close to the ones that are used when it counts to Speech analysis) but also in a quantitative way of analyzing texts. The final results indicate a sensitive lack of agreement about the terminology used, but also an abuse of the representation of the deaths caused by this kind of problem, when there are plenty of other situations caused by this type of violence which are barely showed as that.
\end{abstract}

Keywords: Mass media, gender-based violence, domestic violence, Spanish daily newspapers. 


\section{Introducción}

En la actualidad vivimos un momento de auge con respecto a la concienciación política y social para con la violencia que azota a las mujeres, realidad, que, entre otros términos, ha venido a denominarse "violencia de género". Los medios de comunicación, en ese contexto, gozan de un inestimable poder de influencia social, que, unido a su intrínseca esencia de servicio público, ha llevado a ciudadanos y a instituciones a exigirles un comportamiento responsable y especialmente cuidadoso. El Delegado del Gobierno para la Violencia de Género, Miguel Lorente Acosta se refirió incluso durante las jornadas Los retos de los medios de comunicación ante la violencia de género, celebradas en Valencia en 2009, a la relación existente entre la cantidad de noticias publicadas por los medios y el aumento o descenso de la sensibilidad social con respecto a la violencia de género y las consecuencias de estas oscilaciones en el número de homicidios y asesinatos. "Vemos que conforme la sensibilidad aumenta, al año siguiente disminuyen los homicidios; pero cuando la sensibilidad baja, el año siguiente aumentan los asesinatos. Hay una relación inversa entre sensibilidad y número de homicidios" (Lorente, 2009: 51).

Y no sólo desde el espectro político los medios de comunicación son valorados como entes socializadores y de conocimiento de primer orden. La propia ciudadanía, en una encuesta realizada por el ya extinto Ministerio de Igualdad, afirma conocer este fenómeno, en un 95\% de los casos a través de los medios de comunicación social (Ministerio de Sanidad, Política Social e Igualdad: 2011a). La conclusión a la que se llega es sencilla: la violencia de género será lo que los medios cuenten que es la violencia de género.

No obstante, el poder de los medios no redunda tan sólo en la incidencia de éstos sobre la sensibilidad del cuerpo social. Aspectos como la tematización de la violencia de género, el tipo de encuadre periodístico dado a la información o la terminología de referencia utilizada en las informaciones se configuran como elementos vitales en un análisis concienzudo y posibilista de estos poderosos entes. Las formas de nombrar, caracterizar y representar los diferentes aspectos de la violencia de género influirán tanto en la sensibilidad como en la propia concepción de los públicos sobre qué es la violencia de género, de qué formas puede manifestarse y cuáles son los recursos al alcance de las víctimas. Incluso, recientes estudios ecológicos parecen demostrar que determinados encuadres noticiosos de asesinatos de mujeres influyen en la multiplicación de actos violentos en los días posteriores a la publicación de la información (AAVV: 2009).

Volviendo a la responsabilidad otorgada a los medios, es la propia Ley Orgánica 1/2004 la que en parte de su articulado defiende la necesidad de implicación de los medios de comunicación social como parte de la dinámica de cambio necesario, ya que en su articulado se les insta a salvaguardar la igualdad y a propiciar un trato no discriminatorio: "La difusión de informaciones relativas a la violencia sobre la mujer garantizará, con la correspondiente objetividad informativa, la defensa de los derechos humanos, la libertad y dignidad de las 
mujeres víctimas de violencia y de sus hijos. En particular, se tendrá especial cuidado en el tratamiento gráfico de las informaciones”, capítulo II, art. 14 (España, 2004: 42170).

En todo caso, la información que recibe la sociedad civil es, en primera instancia, la de los medios de información. Por ello, la manera en que éstos discriminan las informaciones, las conceptualizan (con el uso de cintillos o ubicándolos en las distintas secciones) y las desarrollan, tiene una gran importancia desde el punto de vista tanto de la sensibilización como de la formación social. Son los medios los grandes proveedores de información sobre la violencia de género, sobre sus formas de manifestación - y hasta ahora la violencia psicológica o económica se ha tratado poco- sus consecuencias o los recursos disponibles para las mujeres que la sufren. Por todo ello, el esfuerzo exigido a los medios cabe pasar, en primer lugar, por la denominación y la conceptualización en sí, algo que puede parecer nimio pero que esconde, cual punta de iceberg, todo un conglomerado de incertidumbres ideológicas, posicionamientos empresariales y falta de conocimiento sobre la propia temática. Ya que, como defiende Celia Amorós, "conceptualizar es politizar": "los conceptos críticos posibilitan la visibilización de determinados fenómenos que no se visibilizan desde otras orientaciones de la atención y a su vez, esta visibilización nutre y posibilita nuevos conceptos críticos” (Amorós, 2008:17).

\section{Hipótesis y objetivos}

Como dijera Manuela Castro Santiago en La belleza ética de las palabras (2004) este proyecto está guiado por la premisa de que "las palabras constituyen el fundamento de nuestro pensamiento y moldean nuestra forma de sentir y percibir la realidad" (Castro, 2004). La idea principal en la que se sustenta esta investigación es la de que las palabras construyen y modelan nuestras estructuras cognitivas por lo que la elección y precisión de los términos que se utilizan para referirse a los fenómenos han de ser lo suficientemente adecuadas como para que puedan sustentarse en ellas realidades complejas y queden éstas bien definidas.

En este sentido, coincidimos completamente con autores como Guerrero Salazar (2007) quién apela a la gran responsabilidad lingüística y terminológica de los medios de comunicación social en cuestiones tan sensibles como las de género. Para Guerrero Salazar (2007:1) de la Universidad de Málaga,

"los medios de comunicación tienen una gran responsabilidad en la imagen pública de las mujeres que se nos crea a través del lenguaje que utilizan. Este lenguaje puede ocultarlas, discriminarlas e incluso denigrarlas. Por el contrario, un tratamiento igualitario en el discurso mediático puede contribuir, no solo a visibilizarlas, sino a acelerar el avance hacia la igualdad en muchos otros ámbitos".

Apelando a la inexorable responsabilidad que han de tener los media como agentes sociales de primer orden en el campo de la violencia de género (responsabilidad que el legislador recuerda en la Ley Orgánica), partimos de ciertas consideraciones que pasamos a explicitar: 
1) Existe una clara falta de consenso con respecto al término político "violencia de género". Cabe referirse a esta voz como "término político" debido a la ausencia de acuerdo entre los distintos agentes sociales implicados en este asunto. Al ser un término de algún modo "impuesto" políticamente al sancionarse la Ley, terminología legislativa, conceptualización mediática y jerga popular no confluyen en el panorama social. Lo que puede conllevar un desorden cognitivo importante y contraproducente para con una lacra que ha de eliminarse con el esfuerzo de todas las fuerzas sociales.

2) La falta de consenso esconde tanto implicaciones ideológicas (como evidencian los debates surgidos en torno a esta voz) como cognoscitivas. La ausencia de un acuerdo tiene que ver con una falta de convencimiento y de conocimiento, lo que puede conllevar:

2.1) Simplificación temática y caos perceptivo (infravaloración del problema).

2.2) Ausencia de especialización en el medio (tratamiento inadecuado).

2.3) Disminución del beneficio social (de la divulgación de esta clase de noticias).

2.4) Imposibilidad de realizar investigaciones profundas (para analizar, denunciar y mejorar la producción de textos periodísticos sobre el tema).

3) Existe una proliferación de noticias sobre violencia de género que no son encuadradas como tal debido a la consideración de este fenómeno que se produce con determinadas formas lingüísticas. Es decir, una gran cantidad de noticias que tratan el problema de la violencia de género no son identificadas como tal en los periódicos de referencia a nivel nacional. En parte por el desconocimiento de la multiplicidad de manifestaciones de esta violencia, y en parte también por un problema de consenso conceptual.

La hipótesis de este trabajo puede concretarse por tanto, en que a pesar de la evolución positiva sufrida en la visibilidad de este problema y la proyección pública de las noticias de violencia de género, aún pervive una explícita falta de consenso terminológico y una falta de conceptualización en cuanto a las diversas formas de violencia de género relatadas habitualmente en prensa haciendo por tanto los medios de comunicación dejación de sus funciones en cuanto a formadores e informadores sociales. Los objetivos de investigación que nos hemos marcado han sido los siguientes:

1) Reconocer, cuantificar y analizar las formas con que 4 periódicos españoles refieren la "violencia de género".

2) Cuantificar el número de noticias sobre este fenómeno que aparecen en la prensa dado el periodo de análisis y cuáles son reconocidas y explicitadas como tal. 
3) Ahondar, consecuentemente, en el concepto de violencia de género, realizando una comparativa con las definiciones dadas por diversos organismos y la terminología implícita en la configuración de los textos informativos.

4) Relacionar el concepto de violencia de género al uso con las formas más comunes de este tipo de violencia en nuestros periódicos de referencia partiendo de la base de que únicamente lo que se trata como noticia es lo que posteriormente es considerado como violencia de género.

\section{Método}

Para alcanzar los objetivos de esta investigación, aún en marcha, se ha seguido una metodología mixta híbrida, a medio camino entre el análisis crítico del discurso (ACD) (Van Dijk: 1996, 2000, 2003) y el análisis de contenido (AC) con la finalidad de objetivar todo lo posible las referencias tangibles y explícitas denotadas en los productos periodísticos de las que nos provee el AC.

Si bien el espíritu de esta investigación es de tipo crítico y persigue lo que Van Dijk reconoce como "análisis crítico" y que define como una investigación que no persigue necesariamente aplicar un modelo o validar un paradigma sino que adopta un objetivo superior, el de desentrañar los modos en los que el discurso contribuye a la reproducción de la desigualdad y la injusticia (Van Dijk, 1994).

En la misma línea se ha adoptado el AC como método de "desocultación" (Bardin, 1986:7). Este análisis ha sido definido por Krippendorff (2004) como una técnica de investigación que se utiliza para hacer inferencias reproducibles y válidas de los datos al contexto de los mismos y por Bardin (1986:7) como un "conjunto de instrumentos metodológicos cada vez más perfectos y en constante mejora, aplicados a discursos (contenidos y continente) extremadamente diversificados".

Las noticias tomadas como referencia principal (aunque han sido contextualizadas con un centenar de ellas) se circunscriben al intervalo 1- 7 julio de 2010. Al ser nuestra pretensión establecer una mirada analítica sobre un conjunto de hábitos, la extracción de una pequeña muestra de éstos - reflejo de la cotidianeidad de los textos publicados- no es sino un modo de hacer asequible esa realidad para su estudio. Esto halla su razón última al entenderse que se necesita tan sólo una pequeña muestra para comprender un continuum en la labor periodística.

Un segundo razonamiento para la elección de la muestra de referencia es la alta concentración de casos de muertes por violencia de género en los periodos estivales. Los últimos datos del Ministerio de Sanidad, Política Social e Igualdad al respecto apuntan como en el mes de julio la media de mujeres muertas por esta causa desde el año 2003 al 2010 asciende a 6,9; la segunda tasa de mortalidad (mensual) más alta del año. 
Gráfico 1: Víctimas mortales anuales por violencia de género 01/01/2003a 31/07/2011

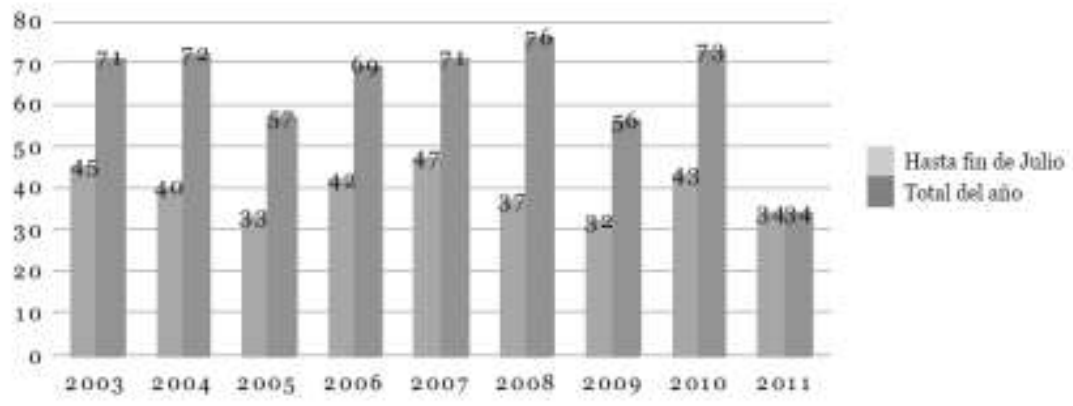

Fuente: Ministerio de Sanidad, Política Social e Igualdad (2011b)

A continuación se expone en una tabla el número de muertes por esta causa desglosado por meses en el que el aumento de los casos durante los meses estivales queda patente. En dicha tabla no se exponen las cifras del año 2010 ya que en el periodo de análisis coincide con la fecha real (se llevó a cabo la recogida de las informaciones al tiempo de sucederse).

Tabla 1: Media estadística de muertes por violencia de género entre 2003-2009

\begin{tabular}{|c|c|c|}
\hline Mes & $\mathbf{N}^{\mathbf{0}}$ muertes (2009) & Media 2003- 2009 \\
\hline Enero & 0 & 5,3 \\
\hline Febrero & 5 & 5,7 \\
\hline Marzo & 4 & 5,1 \\
\hline Abril & 3 & 4,7 \\
\hline Mayo & 9 & 6,0 \\
\hline Junio & 5 & 6,0 \\
\hline Julio & 6 & 6,6 \\
\hline Agosto & 5 & 7,0 \\
\hline Septiembre & 6 & 5,0 \\
\hline Octubre & 6 & 5,4 \\
\hline Noviembre & 0 & 4,9 \\
\hline Diciembre & 5 & 5,3 \\
\hline
\end{tabular}

Elaboración propia. Fuente: Ministerio de Sanidad, Política Social e Igualdad

Cabe destacar que estas cifras sólo hacen mención de los casos cuyo resultado es un homicidio o asesinato, solamente la consecuencia última de este problema, teniendo lugar cada día numerosos y variados episodios de violencia de género. Además, existen multitud de casos en los que la violencia de género permanece invisible, no ya a los ojos de los medios de comunicación, que en ocasiones deciden no publicar estas informaciones, sino a los ojos de la sociedad.

La elección de este periodo de análisis también ha confiado, con resultado positivo al calor de la gran proliferación temática que expondremos, en que ante un mayor número de 
muertes haya una mayor cobertura informativa que permita contar con un corpus informativo lo suficientemente amplio como para no caer en generalizaciones sin fundamento. Es necesario mencionar también cómo este estudio aborda la información, el discurso -su calidad en forma de referente -, sobre violencia de género, no la complejidad de este fenómeno en si mismo.

El corpus de la investigación se ha compuesto por 55 noticias de cuatro diarios distintos referentes a 26 hechos diferenciados. Las informaciones recogidas han sido seleccionadas bajo el criterio de una definición formal de violencia de género incluyente y compleja. Se ha usado como referente para operacionalizar el problema de investigación la definición ofrecida por el principal organismo internacional que ha impulsado la lucha por los derechos de la mujer y contra la violencia ejercida sobre ella: la Organización de Naciones Unidas (NNUU), sin reparar en la definición -o ausencia de ésta- que ofreciera el medio de comunicación ya fuera en su libro de estilo o en la forma de conceptualización del fenómeno en la noticia misma.

Las NNUU han definido en diversas convenciones y resoluciones de la Asamblea General este tipo específico de violencia de manera amplia, inclusiva y contundente. De hecho, ya en el año 1993 la había tipificado como una violación "de los derechos humanos y libertades fundamentales" (Organización de Naciones Unidas, 1993). En ésta se establece que: "por violencia contra la mujer se entiende todo acto de violencia basado en la pertenencia al sexo femenino que tenga o pueda tener como resultado un daño o sufrimiento físico, sexual o sicológico [sic] para la mujer, así como las amenazas de tales actos, la coacción o la privación arbitraria de la libertad, tanto si se producen en la vida pública como en la vida privada".

Además, en el artículo 2 de la misma resolución, se detallan las formas que puede adquirir esta violencia que, por otro lado, conceptualizan como "violencia contra la mujer" y no como "violencia de género". En la resolución se especifica:

"se entenderá que la violencia contra la mujer abarca los siguientes actos, aunque sin limitarse a ellos:

a) La violencia física, sexual y sicológica que se produzca en la familia, incluidos los malos tratos, el abuso sexual de las niñas en el hogar, la violencia relacionada con la dote, la violación por el marido, la mutilación genital femenina y otras prácticas tradicionales nocivas para la mujer, los actos de violencia perpetrados por otros miembros de la familia y la violencia relacionada con la explotación.

b) La violencia física, sexual y sicológica perpetrada dentro de la comunidad en general, inclusive la violación, el abuso sexual, el acoso y la intimidación sexuales en el trabajo, en instituciones educacionales y en otros lugares, la trata de mujeres y la prostitución forzada.

c) La violencia física, sexual y sicológica perpetrada o tolerada por el Estado, dondequiera que ocurra" (Organización de Naciones Unidas, 1993).

Además, como complemento a esta definición internacional se han tenido en cuenta las valoraciones de éste fenómeno que el ordenamiento jurídico nacional hace al respecto y que lo conceptualiza de la siguiente manera:

a) Como una forma de desigualdad.

b) Como un problema público y que afecta -y responsabiliza- a la sociedad en su conjunto. 
c) Como dirigido única y exclusivamente contra las mujeres.

d) Que tiene su origen en la falta de consideración, por parte de los agresores, de los mínimos derechos de libertad, respeto y capacidad de decisión de las mujeres a las que agreden.

e) Que tiene lugar en el seno de las relaciones de afectividad, con o sin convivencia.

f) Que abarca "todo acto de violencia física y psicológica, incluidas las agresiones a la libertad sexual, las amenazas, las coacciones o la privación arbitraria de libertad" (España: 2004: 42168).

Dada la amplitud de esta definición y con la pretensión de exhaustividad que esta investigación requería, no se ha procedido para la recopilación hemerográfica a la asistencia de ningún soporte informático. Es decir, la recopilación de las informaciones acontecidas en el periodo de estudio sobre violencia de género se ha efectuado de manera manual visualizando, leyendo y contrastando con la definición anteriormente expuesta cada una de las noticias de los diarios contemplados.

Los textos incluidos en este análisis se corresponden con el género periodístico informativo denominado "noticia", obviando los géneros de opinión en todo caso y admitiendo dentro de esta categoría noticias ampliadas y piezas cercanas al género del reportaje. Se ha entendido durante todo el proceso la noticia en su ámbito formal (espacio gráficamente delimitado con un contenido noticioso caracterizado por elementos de titulación y firma independiente de otros textos) y no como unidad de sentido. Es decir, a veces, un mismo acontecimiento puede configurarse como dos o más noticias independientes. En ese caso, se contabilizarán el número de noticias, no los hechos que las motivan. Distinguimos por tanto el número de temas o asuntos que aparecen noticiados en el medio del número de noticias que han propiciado.

El criterio principal en todo caso lo ha constituido el carácter informativo y no opinativo de los textos al considerarse en esta investigación que es en los géneros de información en los que el público disminuye sus barreras o reticencias contra la manipulación y tiende a impregnarse de la lógica de la neutralidad de la que se revisten, por diversos mecanismos (uso de la tercera persona, por ejemplo), estos textos informativos. Tampoco se han incluido en este análisis los encartes ni suplementos que, de modo ocasional, pudieran acompañar a los diarios.

Los medios de referencia en este estudio han sido seleccionados siguiendo un criterio de representatividad, tanto desde un punto de vista numérico sobre el índice de lectores en la esfera nacional española como en cuanto a ideología- línea editorial. Por ello se han tenido en cuenta no sólo los diarios generalistas de mayor difusión ${ }^{1}$ sino los que representan las diferentes opciones del panorama ideológico de nuestro entorno. Así, los diarios analizados han sido:

\footnotetext{
${ }^{1}$ Según los datos de noviembre 2010 del EGM los diarios generalistas de mayor audiencia son, en este orden: El País (2.012.000 número de lectores), El Mundo (1.329.000), El Periódico (791.000), Abc (751.000), La Vanguardia (749.000), La Razón (351.000), Público (239.000).
} 
- El País. Referente a nivel nacional e internacional y diario con mayor número de lectores en el panorama mediático español. Se ha autoproclamado hasta hace relativamente poco tiempo "diario independiente" y se le considera el abanderado del progresismo periodístico español.

- El Mundo. Es el segundo diario generalista más leído en España y representa intereses ideológicos contrapuestos al líder de audiencia.

- Público. A pesar de su juventud y de ser el séptimo diario español en número de lectores constituye una referencia en cuanto a implicación en materia de violencia de género, habiendo aparecido en el panorama mediático con un decálogo para informar sobre violencia de género (Público, 2008).

- Abc. Representa en el panorama de medios español una tendencia política eminentemente conservadora.

Para poder acercarnos a los objetivos generales de la investigación se ha elaborado una ficha de extracción de datos original que ha contado entre otras con las siguientes variables de estudio a fin de sistematizar la lógica del discurso mediático sobre la violencia de género, centrándonos, siempre, en el aspecto terminológico y conceptual. Se ha pretendido que esta ficha sea fiel a tres contextos: medios de comunicación, agresiones a mujeres y análisis sistemático del discurso. En ésta se recogen una multiplicidad de variables entre las que destacamos las siguientes:

- Ubicación privilegiada (se han considerado situaciones privilegiadas las de mayor incidencia visual y temática: la portada del diario y la portadilla de sección).

- Sección en la que se encuentra la información.

- Titular (se han recogido todos los elementos que lo componían: antetítulo, título y subtítulo).

- Forma terminológica empleada (estima las múltiples formas en las que los medios estudiados conceptualizan la violencia de género. Dados los estudios realizados con anterioridad y la experiencia periodística se ha optado por una variable multirrespuesta posteriormente recategorizada).

- Ubicación privilegiada de la forma terminológica (para esta investigación que trata de formas de violencia de género y de las formas terminológicas con las que se alude a ésta, la presencia de dichos términos en lugares destacados gráficamente (como titulares, ladillos, cintillos, etc.) constituye un gran valor. Así, pretendemos analizar qué términos referidos a violencia de género se explicitan y publicitan -qué mejor manera que en un titular- abiertamente en la práctica del periodismo con el valor de reconocerse el medio en ellos. Con esta variable se pretende inferir con qué términos se identifica cada medio y cuáles son sus preferencias de uso ya que consideramos que al exhibirlo 
en lugares destacados puede -en relación con otras categorías- ser considerado como el término idóneo para el medio que las utiliza).

- Forma de violencia de género (se contemplan las siguientes en orden alfabético: acoso, agresión física, agresión física a menores, agresiones a hombres, agresión sexual, agresión sexual a menores, agresión psicológica, agresión psicológica a menores, muerte, muerte de menores y tráfico de personas. También se contempla la categoría "otras formas". La inclusión de la variable "agresiones a hombres" es entendida en tanto que, en la lógica del discurso mediático, las noticias de hombres agredidos, ya sea por mujeres o por otros hombres con los que media una relación sentimental, están estrechamente ligados con la proliferación y publicidad de las noticias de violencia contra la mujer).

- Marcadores temáticos.

- Tratamiento como problema global (esta variable refleja si la información recoge el hecho violento al que se refiera la noticia como un problema global, recomendación general en la mayoría de los manuales de redacción de noticias sobre violencia de género, lo que indudablemente requiere una lectura discursiva cualitativa).

\section{Resultados}

"Conceptualizar significa pasar de la anécdota a la categoría y, precisamente, en esta cuestión de los malos tratos y asesinatos de mujeres ha sido enormemente difícil que se produjera este paso. No se producía a causa de un círculo vicioso: las anécdotas eran anécdotas porque no se sumaban, pero a su vez no se sumaban porque eran consideradas anécdotas” (Amorós, 2008: 15).

La más importante de las conclusiones de este trabajo está precisamente en sintonía con lo que la filósofa Celia Amorós seguía planteando en 2008: la importancia de la categorización, de lo que en periodismo se denomina "tematización", ámbito en el que no se está ganando la batalla en los medios de comunicación. Ya en 1997 con el archiconocido episodio de Ana Orantes éstos iniciaron una toma de posición muy clara ante el fenómeno de la violencia contra las mujeres. Empezaron a visibilizarlo y a concienciar sobre este fenómeno, sacando los casos a la luz, luchando por convertirlo, de la mano de los movimientos feministas (Ferrer y Bosch, 2006) en un problema público relegando al ostracismo, en su sentido más ateniense, aquello de "la vida privada" y de "mi marido me pega lo normal". Los medios convirtieron los "dramas familiares" en auténticos casos públicos y concienciaron a la población sobre la magnitud del problema y la responsabilidad de toda la sociedad en su erradicación. En la actualidad, sin embargo, una gran crítica puede hacerse a la gestión informativa que están llevando a cabo ya que, en su afán por visibilizar los dramas personales han configurado una realidad sesgada de la violencia contra la mujer. Lucharon para que dejase de considerarse el maltrato un asunto privado y no han hecho otra cosa que favorecer que no se considere privado, pero que siga relegándose al ámbito de lo doméstico. Alimentando que prevalezcan lo que Carmen Caballero describió muy acertadamente como "víctimas de andar por casa" (Amorós, 2008: 18). Los 
medios no han generado la conciencia en torno a la violencia contra la mujer como un problema social complejo, estructural y poliédrico sentando el conocimiento necesario para comprender el nexo de unión entre la trata de blancas, la mutilación genial femenina y el abuso sexual de adolescentes. Violencia de género sigue siendo hoy lamentable muerte de una mujer a manos de su marido o pareja.

Si bien los medios han facilitado el acercamiento social a esta realidad, ni están sabiendo categorizarla correctamente para crear un marco de sentido propio sobre el problema, ni están "sumando". Con la multiplicidad terminológica de un lado y la ausencia de referencias ${ }^{2}$ por otro están continuando la fragmentación de ese panorama desolador de "casos" que no hace sino contribuir al desconocimiento general sobre el problema deformándolo y elevando una de sus manifestaciones a representante y carácter general del asunto (Zurbano, 2010).

Antes de comenzar a exponer los principales resultados de la investigación vamos a desarrollar gráficamente el corpus de la investigación al que nos hemos referido anteriormente. En el siguiente cuadro se ponen de manifiesto, por un lado, los temas que han aparecido, el número de noticias que ha dedicado cada medio a ellos y las coincidencias y originalidades temáticas de cada uno de los cuatro diarios de referencia. Así, nos encontramos con un panorama mediático prolífico en cuanto a informaciones sobre formas y acontecimientos de violencia de género y con un gran nivel de coincidencia en ciertos temas deliberadamente destacados por estos medios, como por ejemplo el llamado "caso de Pinos Puente", caracterizado por su brutalidad. En total, contamos con 26 ejes temáticos sobre violencia contra la mujer en apenas una semana.

Cuadro 1: relación de temas y noticias por medios

\begin{tabular}{||cccccc||}
\hline \hline $\mathbf{N}^{\mathbf{0}}$ & Hechos/ suceso & Público & Abc & El País & El Mundo \\
1 & Mira Sorvino trata de blancas & I & & & \\
2 & Red explotación & I I & & I & \\
3 & Caso Pinos Puente & I I I I & I I I & I I I & I I I I I \\
4 & Imán de Cunit & I & I & I I & I \\
5 & Indemnización por secuestro & I & & & I \\
6 & Hombre mata a hombre & I & I & I & \\
7 & Diez nuevos crímenes VG & I & & & \\
8 & Abasolo abuso sexual & I & I I & I & I \\
9 & Caso Puerto Santa María & & I & & \\
10 & Abuso a nieta & & I & & \\
11 & Madre defiende a hijo & & I I & & \\
12 & Caso Dutroux & & & I & \\
13 & Abuso imán CNI & & & I & \\
14 & Caso Mari Luz Cortés & & &
\end{tabular}

\footnotetext{
${ }^{2}$ Nos estamos refiriendo a esas noticias de violencia contra la mujer que son presentadas fuera de todo marco de discriminación sistemática ni encuadre temático alguno y donde la referencia a este problema no existe, construyéndose la información sobre la singularidad de un caso excepcional, que, por otro lado, nunca lo es si se toma como una práctica más de un problema sumamente complejo y polifacético.
} 


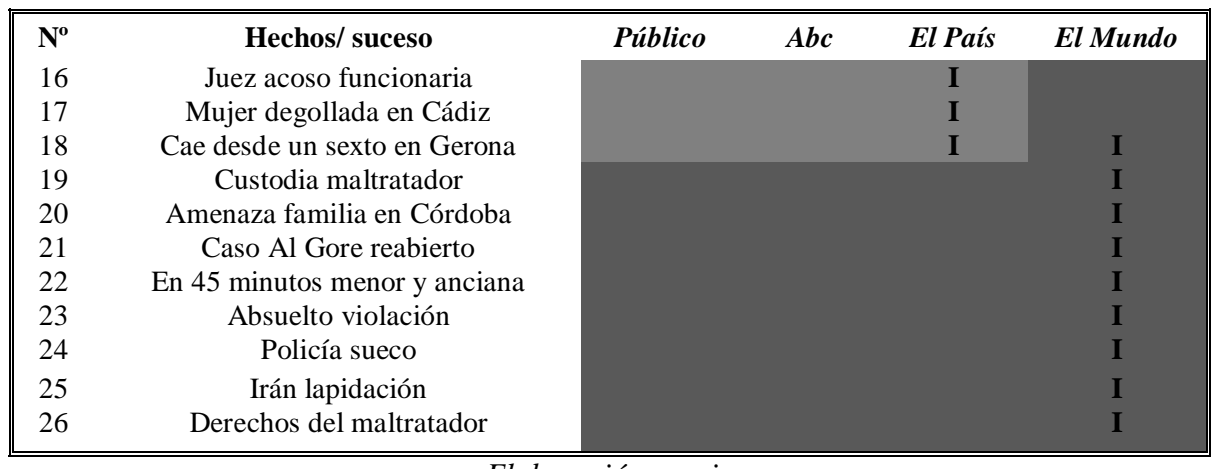

Elaboración propia

La cantidad de noticias dobla al de temas o sucesos acontecidos. A esto, de forma muy sucinta, puede dársele una lectura a priori sencilla: hay sucesos que despiertan un mayor interés periodístico que otros. Como el asesinato de la mujer de Pinos Puente o el caso del imán de Cunit, temas cubiertos por todos los diarios estudiados en los que, además, podemos encontrar más de una noticia al respecto. Es interesante que haya hechos que despierten un especial interés periodístico y es más interesante aún caracterizar las informaciones con más atención mediática: se corresponden con los acontecimientos más escabrosos, dramáticos y con un mayor número de recursos disponibles ${ }^{3}$. En nuestra muestra, el caso más popular es el caso del asesinato "a golpes de azadón"” de una mujer en la localidad de Pinos Puente.

Vicente Romano trata en su obra La intoxicación lingüística (2007) cómo el lenguaje permite la comunicación, la interacción entre los sujetos acercándoles intenciones y situaciones tanto de la parte del emisor como de la del receptor. El lenguaje es la herramienta que posibilita la toma de conciencia, la verificación del conocimiento y el acuerdo en la modificación de las condiciones sociales. Partiendo de esta base, y de lo que Romano, junto a otros, han denominado 'la perversión lingüística', esta investigación se ha preguntado sobre la proliferación terminológica sobre la violencia de género y ha identificado de qué maneras se alude a este problema social.

En Abc, de todas las noticias recogidas y que son configuradas como noticias de violencia de género explicitando alguna de las terminologías al uso ${ }^{6}$ (58,3\%), el 33,3\% contemplan la terminología elegida en el titular y el $75 \%$ pertenecen a la sección de información regional. En el caso de El Mundo las noticias categorizadas como hechos de

\footnotetext{
${ }^{3}$ Nos referimos sobre todo a recursos gráficos.

4 “Asesinada cruelmente a golpes de azadón”. Abc, 2/7/2010: 11.

5 "La perversión se multiplica en mayor proporción que el número de palabras que circulan. Solo el poder tiene la capacidad de dar a la palabras su uso real, a condición de que dé a los hechos su valor real, y no recompense a quienes lo usan para crear víctimas, cárceles, pobres" (Haro Teglen, 2004, en Romano, V. 2007).

${ }^{6}$ Consideramos que los medios se refieren a la violencia de género cuando utilizan alguno de los siguientes términos para referirse a los hechos: violencia contra la mujer, violencia machista, maltrato/malos tratos, violencia doméstica, violencia familiar/intrafamiliar, violencia por razón de sexo, feminicidio. Hemos de matizar que la inclusión de todo este abanico terminológico no significa de manera alguna que se aprueben como sinónimas todas las expresiones aquí recogidas.
} 
violencia de género corresponden al 64,7\%. De las cuales en el 45,4\% de los casos la forma terminológica empleada para encuadrar el suceso como de violencia de género aparece en el titular. El diario El País cuenta con el $42,8 \%$ de sus noticias categorizadas como violencia de género. De éstas, en el 66,6\% de las ocasiones, dicha conceptualización se explicita en el titular. En el caso de Público el porcentaje de noticias categorizadas como de violencia de género asciende al $41,6 \%$ con un $60 \%$ de presencia en el titular. Además, la distribución de noticias de este diario revela una especial concentración en la sección que denomina "Actualidad".

\section{Gráfico 2: porcentaje de conceptualización de las noticias de violencia de género en los medios estudiados}

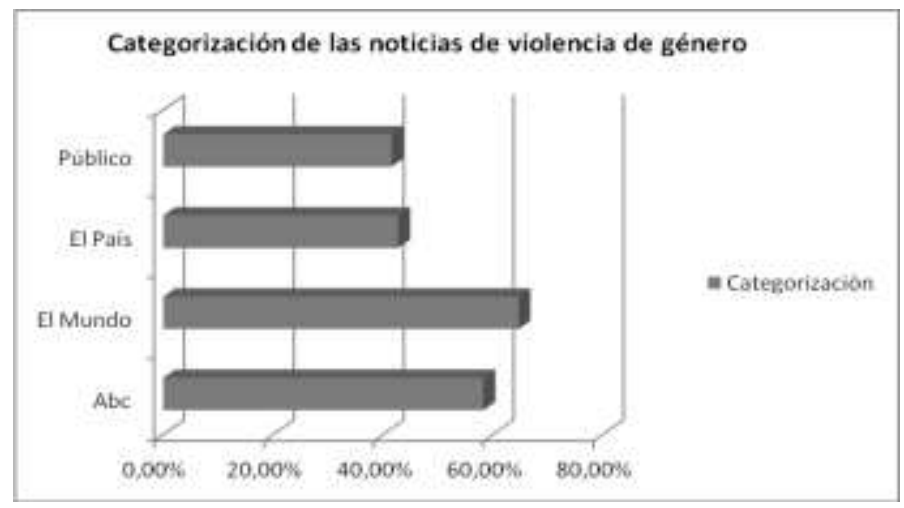

Elaboración propia

Los periódicos que más inciden en el encuadre temático concreto de las noticias de violencia de género son $E l$ Mundo y $A b c$ quienes dotan a las noticias de una terminología que permite enmarcar los acontecimientos en una continuidad temática propia. Sin embargo, esto es así porque los hechos a los que se refieren son en su práctica totalidad muertes y agresiones físicas de un marido/ amante hacia la esposa. Es decir, el patrón clásico de comprensión del fenómeno de violencia de género: la agresión física dentro de la pareja. Si nos remitimos al gráfico inicial en el que se resumían los temas tratados y la concentración mediática en alguno de ellos podemos comprobar cómo estos dos periódicos son los que más atención prestan, y por consiguiente más espacio dedican, a los hechos de Pinos Puente y Santa María.

Además tenemos que señalar que el porcentaje mayor de identificación de los fenómenos como acontecimientos de violencia de género se da en noticias ubicadas dentro de las secciones locales y regionales, espacios dedicados a manifestaciones de violencia de una tipología concreta: la de las agresiones en el seno de la pareja.

Se observan dos pautas periodísticas diferenciadas: por un lado, los diarios con espacios dedicados a lo regional y lo local específicamente y tendentes editorialmente a posiciones

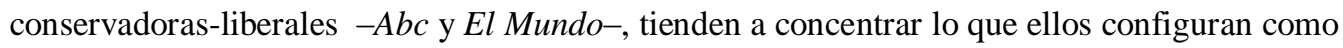
noticias de violencia de género en las páginas dedicadas a la información regional, prefiriendo 
circunscribir el suceso a su ámbito geográfico. Sin embargo, los diarios considerados progresistas y entre cuyas secciones sólo se atiende al ámbito regional, sin ser éste de los espacios más destacados de ninguno de ambos medios, prefieren encuadrar este tipo de noticias en las secciones "de sociedad". A esto hay que añadirle dos salvedades. La que supone el caso de Público, que eminentemente y por encima de la media prefiere encuadrar estas noticias como "de Actualidad" (lo que hemos asimilado como la sección "Sociedad" en otros medios), y la de El País que se debate entre el criterio geográfico (acercándose a la posición tradicional llevada a cabo por el resto de diarios de la muestra) y el de temática más "social".

Por otro lado, es conveniente reseñar cómo durante el periodo estudiado hay ciertos asuntos que - de manera generalizada en los cuatro periódicos- no han sido encuadrados como informaciones de violencia de género: los delitos sexuales. En todos los diarios durante el periodo de análisis se han producido de una a varias noticias sobre delitos sexuales y en ningún caso se han categorizado los actos cometidos como una agresión contra la mujer enmarcada dentro del panorama general de la violencia de género como sí establece por ejemplo la definición de las NNUU (Organización de Naciones Unidas, 1993). Ante esto no cabe sino el análisis en torno a por qué existe un alto porcentaje -variable según cada medio pero oscilando entre el $58 \%$ y el $35 \%$ - de informaciones de este tipo de violencia que no quedan encuadradas como tal. Conviene no obstante no tomar estos datos como absolutos ya que cobran sentido cuando están relacionados directamente con la repetición noticiosa de determinados asuntos. Es decir, en el caso de $\mathrm{El}$ Mundo, diario con un porcentaje menor de noticias no categorizadas como de violencia de género, se da la coyuntura de que es el que mayor número de noticias publica sobre el caso de la muerte de Pinos Puente que es definido por todas las cabeceras como un "crimen de género" o "machista", lo que tiende a incrementar su porcentaje de noticias explícitamente encuadradas como de violencia de género.

Con respecto a la ubicación de las noticias, diarios tan dispares ideológicamente como $A b c$ y Público coindicen. Si antes ya lo hicieron en la manifestación del interés y las consabidas reiteraciones noticiosas con respecto a asuntos como el crimen de Pinos Puente, ahora lo harán en la situación preferente que dan a las noticias de violencia de género en las páginas impares. Siguiendo a Hernández Guerrero (2009: 4): “El orden de las páginas establece una jerarquía de importancia informativa [...] las páginas impares poseen mayor valor que las pares ya que sobre ellas recaen espontáneamente las primeras miradas".

Tabla 2: Situación de las noticias de violencia de género en las páginas de los diarios estudiados

\begin{tabular}{ccc}
\hline Medios & Páginas pares & Páginas impares \\
\hline Abc & $25 \%$ & $75 \%$ \\
El Mundo & $52,9 \%$ & $47 \%$ \\
El País & $71,4 \%$ & $28,5 \%$ \\
Público & $41,6 \%$ & $58,3 \%$ \\
\hline \multicolumn{3}{c}{}
\end{tabular}


No sólo la ubicación de las noticias en el periódico nos indica a qué criterios de selección está sometida dicha noticia sino que es fundamental también la sección en la que ésta se inserta ya que será ahí dónde el medio refleje -si existe homogeneidad de trato o de no existirla revelará otros matices a estudiar- a qué categoría noticiosa relaciona el hecho. Los principales resultados obtenidos en nuestra muestra son los que abajo se reflejan y dan como resultado fundamental, como ya hemos anotado con anterioridad, una condensación de informaciones en la sección regional ( $A b c$ y $E l$ Mundo) y en la denominada "Sociedad" o "Actualidad" (El País y Público respectivamente). En el caso de los diarios que observamos prefieren no asociar las noticias de violencia de género a su origen geográfico es destacable que no cuentan entre sus secciones habituales con páginas locales. Es decir, la violencia de género pasa por ser un hecho al que se acerca la información por su proximidad geográfica y que destaca como tal en su sección local, en el mejor de los casos y como práctica habitual. Cuando no es así, los criterios periodísticos actuales despojan a estas informaciones de todo carácter de relevancia y urgencia relegándolos a las páginas, situadas al fin del diario, las de "sociedad".

Conjunto de gráficos 3: Distribución por secciones de las noticias de violencia de género
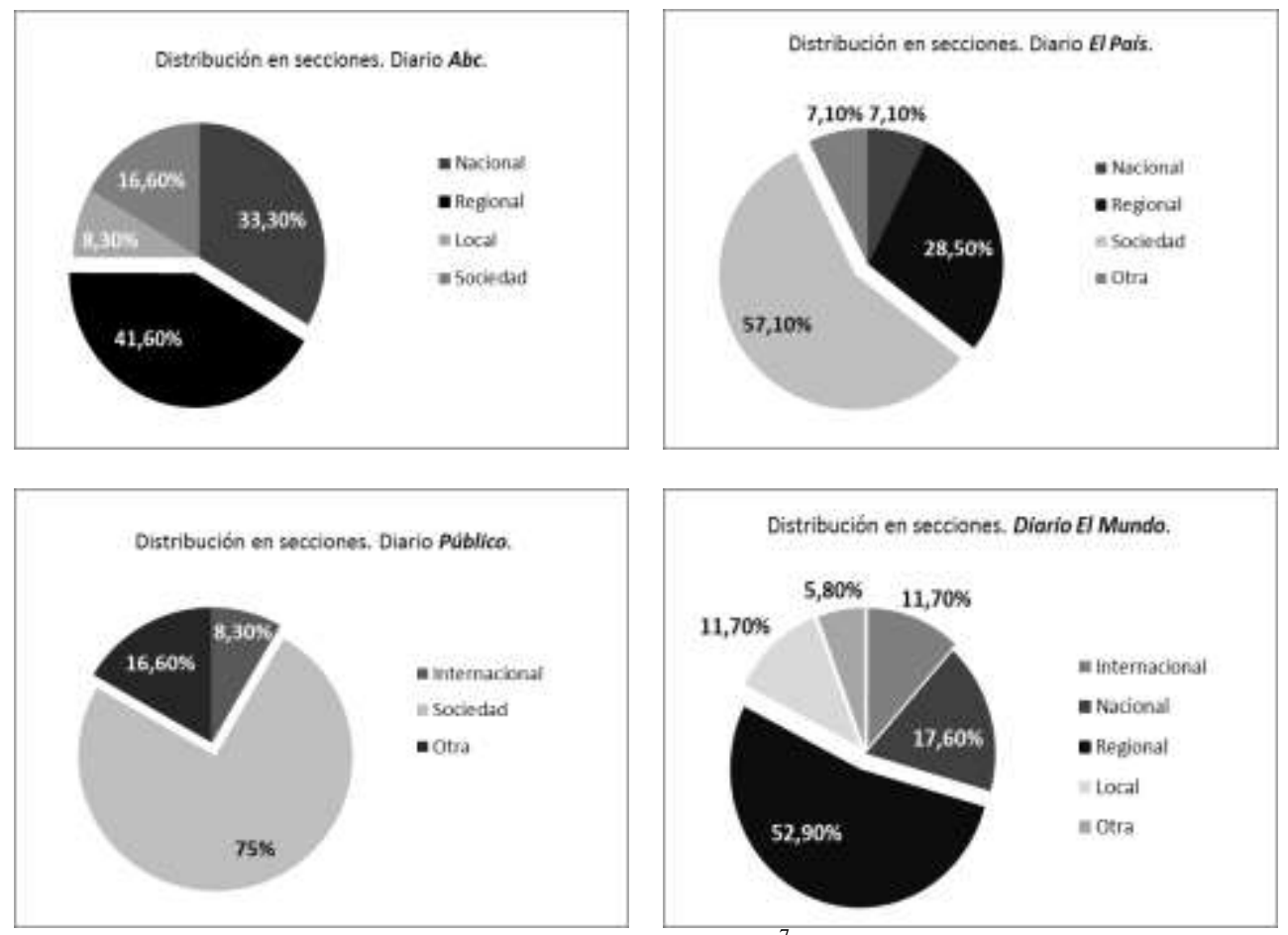

Elaboración propia

\footnotetext{
${ }^{7}$ Las secciones nacional y sociedad en los diarios Público y El Mundo respectivamente no aparecen reflejados en los gráficos por ser nulo el resultado informativo encontrado en dichas secciones.
} 
Sin embargo, que exista esa variedad y esa distribución de las noticias no implica como sosteníamos en la hipótesis inicial- que tales actos sean categorizados y conceptualizados periodísticamente como actos de violencia de género. Sin entrar en un nivel más profundo de análisis redaccional e implícitos latentes, un mero recuento de la forma terminológica con la que se identifican los acontecimientos viene a dar cuenta de que muchas de estas noticias pasan a las páginas de los diarios como meros acontecimientos violentos sin relación entre sí ni encuadre temático alguno. Es decir, sin otro encuadre periodístico diferente al de los sucesos y categorizadas como homicidio, agresión, abuso o "muertes por arrojo desde sexto piso". Es lo que algunos autores han llamado "encuadre episódico" (Iyengar, 1991; Carballido González, 2009).

A fin de profundizar algo más en la no definición de los actos de violencia como formas de violencia de género hemos comenzado en esta investigación, y a modo exploratorio, por relacionar el tipo de violencia al que se hace referencia con su categorización o no por parte del medio. Los tipos de violencia que hemos definido proceden de una re-categorización inspirada por un lado en la definición y tipología de violencia de género efectuadas por las NNUU (que trasciende ampliamente lo que periodísticamente se entiende y trasladada como 'violencia de género') y, por otro, de la experiencia investigadora desarrollada (González y Zurbano, 2010). Dadas las dificultades que existen para analizar de forma cruzada ambas categorías hemos de aclarar algunas cuestiones a fin de hacer lo más legible posible y en un marco de exactitud los siguientes datos.

En primer lugar, es necesario aclarar que generalmente concurren varias formas de violencia contra la mujer simultáneamente por lo que sería tremendamente costoso medir la relación entre las formas de violencia presentadas en la prensa y su categorización o no como formas de violencia dentro del marco de las agresiones a mujeres. De ser estrictamente fieles a la realidad no sólo nos encontraríamos con el problema "técnico" de las variables de respuesta única, o sea, variables cerradas sino que, además, asimilaríamos relato periodístico o relato público (no todos los casos ni sus detalles ven la luz) a la realidad de lo acontecido. Ante una noticia de muerte, por ejemplo, no tienen por qué ser relatados los episodios anteriores de violencia psicológica. Por ello, y a fin de respetar que el marco que nos encontramos es estrictamente periodístico -tratamos con objetos manufacturados no con la realidad misma -, hemos optado por establecer de cada noticia la forma de violencia de género que prevalezca. A lo que seguidamente hemos de añadir que: en el caso de muertes, ésta ha sido considerada la forma principal; en el caso de agresiones a hombres las formas de violencia ejercidas han pasado a un segundo plano atendiéndose al propio relato de la masculinidad como foco principal de atención mediática; en el caso de la violencia psicológica, por tener una menor visibilidad, se la ha considerado cuando se contemple en el relato periodístico forma principal de violencia. En otros casos de evidenciación de más de una forma de violencia en el relato periodístico se ha atendido a la forma destacada en el titular (contemplando todos sus elementos posibles) o en los siguientes elementos gráficos (en este orden de preferencia): destacado, despiece, ladillo. 
Gráfico 4: Aparición de diferentes formas de violencia de género

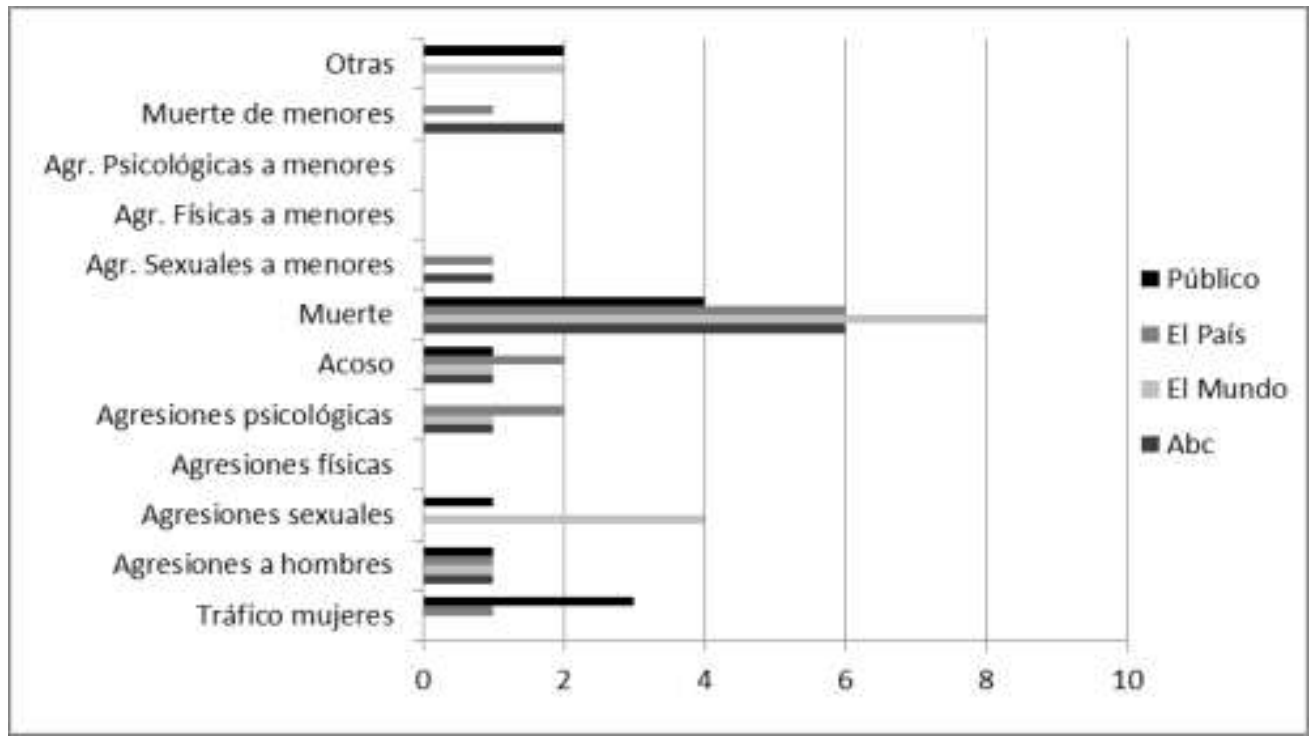

Elaboración propia

Como puede apreciarse en la gráfica anterior, las formas de violencia contra la mujer o violencia de género más visibles en el periodo analizado son los casos de muerte, seguidos de las agresiones sexuales y el tráfico de mujeres. Es destacable, además, el modo en que se comportan estos diarios de referencia en cuanto a la agresión que se produce en Alicante en la que un hombre mata a su pareja, otro hombre. Todas las cabeceras cubren el acontecimiento con una única noticia, pero todos lo cubren. Lo más significativo es que mientras que $A b c$ lo define en el antetítulo de la información como "violencia doméstica" ninguno de los restantes diarios alude a categorización alguna y relatan el hecho sin más.

Con respecto a la vinculación entre la forma de violencia relatada y su inclusión dentro del fenómeno de la violencia de género, hemos comprobado cómo la muerte de menores, los delitos sexuales a menores, y el acoso y el tráfico de mujeres, en ningún caso ha tomado ninguna forma terminológica que los encuadre dentro del fenómeno global de la violencia de género. En el caso del homicidio de la pareja homosexual es únicamente -como exponíamos con anterioridad- el diario $A b c$ el que lo denomina como "hecho de violencia doméstica". De otra parte, las agresiones sexuales se han atribuido a actos de violencia de género solamente en un $40 \%$ de los casos y por concurrencia de varias formas de violencia de género de la que se relata de manera central la agresión sexual. Sin embargo, en las noticias de muerte (24 noticias generadas) en el 91,6\% de las ocasiones se ha atendido a denominar el hecho como una cuestión de violencia de género. Evidenciando el caos conceptual existente -y reproduciéndolo- en el periodismo español en materia de materia de violencia de género, donde terminología, concepto y realidad se simplifican y confunden con tragedias aisladas del ámbito doméstico. 
Finalmente, y como colofón a nuestro objetivo primordial, hemos observado que las preferencias terminológicas para la definición de lo que a nivel mediático se considera "violencia de género" oscilan entre la voz "maltrato", la más utilizada, seguida de "violencia machista" y de "violencia de género". "Violencia doméstica" ha sido utilizada en 4 ocasiones frente a las 21 de la voz "maltrato" o las 13 de "violencia de género" y las 15 de "violencia machista". Por medios, $A b c$ ha usado en el periodo de análisis el término "violencia machista" con mayor profusión, mientras que El Mundo, El País y Público se han centrado en "maltrato". En ninguna ocasión ni en ninguno de los medios analizados se ha utilizado la expresión "violencia contra la mujer".

\section{Discusión}

La forma en la que los medios conceptualizan la violencia de género está ligada a la sobrerrepresentación de la violencia física (incluimos tanto las muertes como las agresiones físicas y sexuales) por encima de otras formas y prácticas como las psicológicas, las económicas o las que son cometidas por los Estados. Ejemplos de ésta última tenemos la acontecida el día 6 de julio y que es tratada por el diario El Mundo: "(Irán) Condenada a lapidación por un delito de adulterio" sin viso alguno de estar relacionado contra los derechos humanos y la violencia que sufren las mujeres sino como una tropelía más de las que se suceden en el panorama internacional, sobre todo en ese tipo de países criminalizados mediáticamente. Esta información, como otras, adopta un encuadre de sucesos centrándose en las vicisitudes del caso que se relata y descontextualizando por completo el problema global femenino -y social- en el que se inserta este hecho. Incluso, en las ocasiones en las que además de violencia física se dan manifestaciones de agresión psicológica ${ }^{8}$ se establece como foco principal lo físico?.

Existe por tanto cierta opacidad en el panorama periodístico español en cuanto a determinadas manifestaciones violentas contra la mujer, circunscribiendo estos actos a los actos físicos que suceden dentro del ámbito de las relaciones íntimas. Si bien en la visibilización y concienciación como problema social, no privado, los medios han realizado una encomiable labor y han demostrado una actitud proactiva, en estos momentos están anclando el problema de la violencia de género a una sola de sus prácticas: el maltrato físico de las esposas. Con las graves consecuencias que ello conlleva, como por ejemplo, la distorsión cognitiva que se produce en los jóvenes. Datos de un estudio del Ministerio de Sanidad,

\footnotetext{
${ }^{8} \mathrm{Abc} 7 / \mathrm{VII} / 2010$ "La madre defiende al joven que mató a su padre y dice que se jugó la vida por ella"; El Mundo 2/VII/2010 "Le piden diez años por maltratar y amenazar de muerte a su ex esposa y a sus hijos en Córdoba"; El País, 3/VII/2010 "Mel Gibson vuelve caer en el pecado de la ira / El actor y director insulta a su ex novia y a los negros"; El País, 5/VII/2010 "Condenado un juez por su "brutal crueldad" contra una funcionaria".

${ }^{9}$ Hecho que, como ya explicamos con anterioridad, nos llevó a primar como "forma de violencia de género" la psicológica en el caso de concurrencia porque en ningún caso hemos encontrado una información sobre casos específicos de violencia psicológica o centrados en ésta.
} 
Política Social e Igualdad (2011c) revelaban el año pasado que existen una multiplicidad de prácticas violentas que los jóvenes españoles no identificaban como tal. Por ejemplo, el $23 \%$ de los chicos y el $14 \%$ de las chicas no consideran una forma de maltrato hacer sentir miedo a su pareja. Si a esto le sumamos el dato de que el $90 \%$ de la población española conoce este problema mediante los medios de comunicación podemos inferir la gran influencia y la responsabilidad de los media sobre el concepto mismo de esta violencia y la consiguiente deformación conceptual que están provocando.

Simplificando y descontextualizando de este modo un problema que es global, atemporal y universal la prensa incurre en una deformación del propio problema ya que atendiendo a los valores de representación que inferimos de la muestra analizada la violencia de género se trata preponderantemente de una agresión física con resultado de muerte y que se da en un contexto familiar, entre cónyuges. Lo que parece estar muy distante de los objetivos que como formadores de la opinión pública poseen los medios de comunicación social, máxime en este problema de especial preocupación social.

La autora de este trabajo quiere agradecer especialmente la colaboración inestimable de Luis Navarro Ardoy (Universidad Pablo de Olavide, Sevilla) en la elaboración de este artículo.

\section{BIBLIOGRAFÍA}

- Amorós, Celia (2008): “Conceptualizar es politizar”. En Laurenzo, Maqueda y Rubio, (coords): Género, violencia y derecho. Valencia: Tirant lo Blanch, pp. 15-26.

- AAVV (2009): "The effect of television news items on intimate partner violence murders". En European Journal of Public Health, Vol. 19, nº 6, pp: 292-596.

- Bardin, Laurence (1986). Análisis de contenido. Madrid: Akal.

- Carballido González, Paula (2009): "Medios de comunicación social y violencia de género. Una revisión desde la teoría del framing". En Bernardo Paniagua, Martínez García y Montiel Roig (coords.): Retos de la Comunicación ante la Violencia de género. Marco jurídico, discurso mediático y compromiso social. Barcelona: Tirant Lo Blanch, pp. 157-176.

- Castro Santiago, Manuel (2004): "La belleza ética de las palabras". En Revista Iberoamericana de Educación, [en línea] Disponible en: http://www.rieoei.org/deloslectores/622Castro.pdf [09/01/2011].

- España (2004): "Ley Orgánica 1/2004, de 28 de diciembre, de Medidas de Protección Integral contra la Violencia de Género". En Boletín Oficial del Estado, 29 de diciembre de 2004, núm. 313, pp. 42166-42197.

- Ferrer, Victoria A. y Bosch, Esperanza (2006): "El papel del movimiento feminista en la consideración social de la violencia contra las mujeres: el caso de España", [en línea] Disponible en: http://www.mujeresenred.net/spip.php?article881 [09/01/2011]. 
- González, Rafael y Zurbano, Belén (2010): "Formas de representación y tratamiento discursivo de la violencia de género en prensa". En Actas del II Congreso de Investigación y Género. Unidad de Igualdad. Universidad de Sevilla.

- Guerrero Salazar, Susana (2007): "El lenguaje sexista en los medios de comunicación”, [en línea] Disponible en: http://www.nodo50.org/mujeresred/IMG/pdf/susana_salazar.pdf [11.12.2011]

- Hernández Guerrero, José Antonio (2009): “Oratoria y periodismo”, [en línea] Disponible en: http://www.biblioteca.org.ar/libros/134849.pdf [11/12/2011].

- Iyengar, Shanto (1991): Is anyone responsible? How television frames political issues. Chicago: The University of Chicago Press.

- Krippendorff, Klaus (2004): Content analysis: an introduction to its methodology. California: Sage.

- Lorente Acosta, Miguel (2009): “¿Qué papel pueden tener los medios de comunicación de masas en la erradicación de la violencia de género al amparo de la ley integral?". En Bernardo Paniagua, Martínez García y Montiel Roig (coords.): Retos de la Comunicación ante la Violencia de género. Marco jurídico, discurso mediático y compromiso social. Barcelona: Tirant Lo Blanch, pp. 157-176.

- Ministerio de Sanidad, Política Social e igualdad (2011a): "III Informe anual del observatorio estatal de violencia sobre la mujer. Informe ejecutivo", [en línea] Disponible en: http://goo.gl/O6Q9K [11/12/2011].

. (2011b): Información estadística de violencia de género. Ficha estadística de víctimas mortales por violencia de género. Julio 2011.

- Ministerio de Sanidad, Política Social e igualdad (2011c): "Igualdad y prevención de la violencia de género en la adolescencia", [en línea] Disponible en: http://goo.gl/PXRCd [11/12/2011]

- Organización de Naciones Unidas (1993): "Declaración para la eliminación de la violencia contra la mujer" (Resolución 48/104 de la Asamblea, en la 85 a sesión plenaria), [en línea] Disponible en: http://www.un.org/documents/ga/res/48/a48r104.htm [30.03.2011].

- Romano, Vicente (2007): La intoxicación lingüística. Barcelona: El Viejo Topo.

- Van Dijk, Teun A. (1994): "Discurso, poder y cognición social”. En Cuadernos, n 2, [en línea] Disponible en http://www.discursos.org/Art/Discurso,\%20poder\%20y\%20cognici\%F3n\%20social.pdf [11.12.2011].

. (1996): “Análisis del discurso ideológico". En Versión, n 6, pp. 15-43.

. (2000): "El discurso como interacción social". En Teun A. Van Dijk (coord): El discurso como interacción social: estudios del discurso, introducción multidisciplinaria. Barcelona: Gedisa, pp. 19-66.

(2003): "La multidisciplinariedad del análisis crítico del discurso: un alegato a favor de la diversidad”. En Ruth Wodak y Michael Meyer: Métodos de análisis crítico del discurso. Barcelona: Gedisa, pp. 143-177.

- Zurbano Berenguer, Belén (2010): “El uso del concepto ‘violencia de género’ en la prensa diaria de tirada nacional en España". En Actas del II Congreso Internacional Latina de Comunicación Social (Tenerife 7-10 diciembre 2010), [en línea] Disponible en: http://www.revistalatinacs.org/10SLCS/actas_2010/Zurbano_genero.pdf [11/12/2011]. 